\title{
Inscripciones curriculares del pensar situado en Trabajo Social
}

\section{Apuntes para una investigación otra en torno a la formación profesional}

Curricular inscriptions of situated knowledge in Social Work Notes for another research on graduate training

María Eugenia Hermida

Licenciada en Servicio Social

(Universidad Nacional de Mar del Plata)

Especialista en Docencia universitaria

(Universidad Nacional de Mar del Plata)

Doctora en Trabajo Social

(Universidad Nacional de Rosario)

Co-directora de la Maestría en Políticas Sociales y del Proyecto de investigación sobre Feminismos del Sur e Intervención social

(Universidad Nacional de Mar de Plata).

Docente-Investigadora

Correo: mariaeugeniahermida@yahoo.com.ar
Renzo Tiberi

Licenciado en Trabajo Social

(Universidad Nacional de Rosario)

Docente en Facultad de Ciencia Política y RRII (Universidad Nacional de Rosario)

Correo: renzotiberi4@gmail.com

Yanina Roldán

Licenciada en Servicio Social

(Universidad Nacional de Mar del Plata)

Especializanda en Métodos y técnicas de Investigación Social (CLACSO)

Becaria sistematización

(Universidad Nacional de Mar del Plata)

Correo: roldanyanina12@gmail.com 


\title{
Resumen
}

En el siguiente artículo presentamos la categoría-concepto de inscripciones curriculares, basada en experiencias previas de investigación en torno a la formación de grado en Trabajo Social. Para ello, partimos de explicitar los alcances de la noción de "investigación otra". Luego trazamos una genealogía colectiva sobre dichas experiencias, que, desde nuestro punto de vista, sirvieron de fundamento, justificación y antecedente en la construcción de la mencionada categoría. Comenzamos con la discusión que Hermida (2016) propuso en torno a los discursos sobre Estado, Poder y Política en la formación académica en Trabajo Social en la Argentina. Esta investigación es significativa considerando que los aportes teóricos, epistemológicos y metodológicos surgidos ahí, orientaron las otras investigaciones. Continuamos con las contribuciones de la tesis de grado de Tiberi (2018), en la que analiza el pensamiento latinoamericano en la currícula de la formación de grado de Trabajo Social en la Universidad Nacional de Rosario. Luego, presentamos la problemática de los Feminismos del Sur en la formación profesional, a partir de la tesis de Roldán (2020), que se desarrolló en la Universidad Nacional de Mar del Plata. Estas últimas dos investigaciones devinieron en la reformulación de los problemas de investigación que serán abordados a partir de su estudio a nivel nacional. En este sentido, lo compartido por estas tres experiencias es la pregunta por las vertientes del pensar situado en Trabajo Social.

\section{Palabras clave}

Inscripciones curriculares, Pensar situado, Investigación social, Trabajo Social.

\begin{abstract}
This paper presents a category-concept that we called "curricular inscriptions". It based on the experience of research on the graduate training in Social Work. For that aim, we start by explaining the scope of the notion of "other research". After which we show a genealogy with our experiences, that contributed to constructing the research problem around profesional training. We start with the discussion around the discourses about State, Power and Politics in the bachelor's degree of Social Work in Argentina. That research is very important because is the $\mathrm{PhD}$ thesis that founded the theoretical, epistemological and methodological guidelines that guided the other research. We continue with some contributions of the degree thesis by Tiberi, who analyzes Latin America thought included in the curriculum of the degree training at the Universidad Nacional de Rosario. Then, we present the degree thesis by Roldán, in that work she explicit the issue of the Southern Feminism in the graduate program. So, the similitudes between those experience is to thought in the different meaning of situated knowledge.
\end{abstract}

\section{Keywords}

Curricular inscriptions, Situated knowledge, Research, Social Work. 


\section{Presentación}

Este escrito surge de experiencias de investigación orientadas a la producción de conocimiento relativo a los procesos de formación de grado en Trabajo Social. Investigaciones que revistieron el carácter de tesis de grado y posgrado, a la vez que se enmarcaron en procesos de construcción colectiva de saberes otros en el marco de nuestros proyectos y grupos de investigación.

Estas experiencias que hemos atravesado nos invitan a reflexionar y repensar el ejercicio de la investigación en nuestra disciplina, permitiéndonos afirmar que en todo proceso investigativo se ponen en jaque un conjunto de máximas epistemológicas y metodológicas. Es que las experiencias que desde la producción de conocimientos revisitamos, deconstruimos, pero también habitamos y buscamos transformar, interpelan en más de una ocasión el modus operandi clásico que gobierna el mundo de la academia. Interpelaciones que resuenan, que tensionan, y que nos proponen asumir un compromiso de transformación.

Siempre comenzamos con preguntas. Las preguntas nos mueven, están atravesadas por una mezcla muy potente de deseos personales y de problemas colectivos que van, poco a poco sedimentando en aquello que llamamos "la formulación del problema de investigación". Y cada problema desafía nuestros supuestos, categorías y métodos. Pone al descubierto la fuerza de algunos conceptos, pero también los límites que nos arrojan al querer suturar esa distancia entre la pretensión de generalización de nuestras categorías, y la precisa textura de esa porción de lo social que queremos interrogar. Allí comienzan las incomodidades, las búsquedas desesperadas y apasionadas, las intuiciones, las lecturas de madrugada, los debates... hasta que en un momento coagula un interrogante que se nos aparece como camino posible para avanzar en nuestra tarea y nos invita a transitarlo.

Queremos compartir algo de ese viaje, de esos caminos. De las preguntas y búsquedas que hemos compartido a partir de interesarnos por saber algo más sobre qué se enseña en la formación de grado en Trabajo Social. Nos hemos preguntado qué discursos circulan en torno al Estado, el Poder y la Política en la formación de grado de nuestra disciplina en el país (Hermida, 
2016) y nos queremos preguntar qué se enseña en torno de lo descolonial (Tiberi, 2018) y a los feminismos del Sur (Roldán, 2020) en la formación de grado en el país. En todos los casos hay una plataforma común, que explicitamos que es el supuesto de que hay un conjunto de perspectivas de profundo valor epistémico, estratégico y político para el Trabajo Social Latinoamericano, y cuya circulación es escasa cuando no inexistente. Tiberi (2019) recupera los aportes del pensamiento latinoamericano y pone su mirada en las producciones del giro decolonial. Roldán (2020), por su parte, recoge las contribuciones de los feminismos del Sur. Finalmente, Hermida (2016) las teorías políticas posestructuralistas y des/pos coloniales para pensar un Estado otro. La pregunta por la presencia y las ausencias de estos enfoques es un punto de partida en el horizonte de contribuir a una formación con perspectiva crítica y transformadora, situada en la realidad y los problemas actuales.

Estas experiencias investigativas pasadas y por venir no fueron sencillas. Un conjunto de problemas necesitó ser sorteados. Obstáculos que nos convocaron a la tarea artesanal de hacer y rehacer, ver y rever, construir y deconstruir en el proceso. Sólo el trabajo colaborativo nos permitió avanzar, y es la certeza en la que nos apoyamos para seguir transitando. Por eso queremos compartir este escrito, para hacer circular las herramientas que fuimos forjando, que son limitadas y perfectibles, pero a la vez muy flexibles y maleables. Por lo que entendemos que tienen la posibilidad de aportar alguna pista a otres colegas que caminan por rutas similares a las nuestras y, por qué no, incentivar a aquelles que se han dejado seducir por estos enfoques y ven en ellos una potencia. A la vez, es una forma de recuperar voces críticas que, haciendo una lectura de nuestro artículo, nos regalen sus señalamientos constructivos para detectar los sesgos o limitaciones que nuestra propuesta pueda contener.

Esta es, entonces, una síntesis de la genealogía que nos llevó, en medio de comunicaciones virtuales a las que la distancia y la pandemia nos confinaron, a acuñar el término de inscripciones curriculares. Esta suerte de concepto-herramienta tuvo y tiene el objetivo de dotar de mayor potencia y factibilidad nuestros esfuerzos investigativos en torno a las presencias y ausencias del 
pensar situado (permitiéndonos a su vez focalizar en los feminismos del Sur y/o en lo descolonial según el caso) en la formación de grado en Trabajo Social. Antes de embarcarnos en desmenuzar los fundamentos y alcances de la noción de inscripción curricular, queremos explicitar en qué sentido la misma a nuestro entender facilita lo que hemos dado en llamar una investigación otra.

\section{La apuesta por una investigación otra}

Como bien se referencia en el título de este trabajo, nos situamos en la apuesta por una investigación otra ¿A qué nos referimos cuando asumimos esta posición? En principio vale decir que nos insertamos en una conversación académica que nos antecede y de la que nos nutrimos. Vienen siendo múltiples los esfuerzos por preguntarse en clave epistemológica y metodológica qué supone investigar desde la opción feminista y descolonial. Diverses autores comparten estos interrogantes, buscando desarmar no sólo un conjunto de premisas teóricas que significan el mundo, sino un conjunto de prácticas, dispositivos que reproducen las formas de habitar la academia y de construir conocimiento.

Haciendo un apretado estado de la cuestión relativo a la pregunta por una investigación otra en el campo educativo, encontramos a Ortiz Ocaña (2018), quien nos propone una metodología otra, que supone pasar de un hacer decolonial a un proceso decolonizante. Así, trascendiendo la pregunta por el repertorio de técnicas, apuesta por tres criterios/principios/acciones/ huellas otras: el observar comunal, el conversar alternativo y el reflexionar configurativo.

Desde el registro poscolonial y feminista, De Oto y Alvarado (2017) ubican a las metodologías de investigación en contexto, focalizando en los problemas de la investigación socio-educativa, poniendo a disposición

(...) claves epistémicas para revisar las condiciones de producción y de legitimación del conocimiento, su extensión y circulación, las formas en las que nuestras lecturas (muchas veces sesgadas) impactan sobre los materiales concretos de investigación y los modos en los que algunas categorías obturan la experiencia de pensar ciertos problemas (De Oto y Alvardo, 2017:12). 
Estos elementos no son en absoluto menores: ¿qué categorías nos impiden pensar? ¿dónde circularán nuestras producciones? ¿qué criterios operan para legitimar los resultados de nuestros estudios? Imposible pensar los contornos de una investigación otra sin interrogarse por estas cuestiones.

Desde España, Rivas Flores comparte los sentidos que asocia a la idea de investigación otra en el campo educativo, afirmando que la misma supone plantear

(...) otras formas de expresar y representar los relatos alternativos de realidad, que rompan con la hegemonía de la palabra escrita como vía única y exclusiva de canalizar el saber. El arte en sus diferentes manifestaciones (...) se desarrollan como formas diferentes de narrar, que a su vez implican contenidos diferentes (2020:27).

Vemos entonces que la invención de una investigación otra no sólo remite a renovar las formas de diseñar y transitar el trabajo de campo, o el análisis de los datos, sino también a las derivas del producto de nuestros procesos de construcción de conocimiento. Aquí, la crítica del logocentrismo y la apuesta a una estética anticolonial (Rivera Cusicanqui, 2018) tienen un lugar destacado.

Queremos señalar que estas discusiones contemporáneas en torno de qué es (y qué no) una investigación otra, remiten a nuestro entender a un conjunto de movimientos epistémicos que nos interesa subrayar. Ensayando una sintética cartografía, podemos decir que la idea de una investigación otra tributa a un conjunto de metáforas que Mignolo (2008) propone a la hora de explicar qué se entiende por pensamiento de-colonial así como su reformulación en opción de-colonial. Él nos va a hablar de:

(...) el pensamiento que se desprende y se abre (...) a posibilidades en-cubiertas (colonizadas y desprestigiadas como tradicionales, bárbaras, primitivas, místicas, etc.) por la racionalidad moderna montada y encerrada en las categorías del griego y del latín y de las seis lenguas imperiales europeas modernas (italiano, castellano, portugués, inglés, francés y alemán) (2008:250). 
Lo que nosotres entendemos por investigación otra, es precisamente una experiencia que se abre a ese mundo otro, encubierto, que no nos es aprehensible desde las categorías de la ciencia hegemónica. En esta línea, Mignolo también hace uso de la metáfora del pensamiento fronterizo, en tanto "método del pensamiento y la opción de-colonial" (2008:252). Nos advierte así de la necesidad de duelar la pretensión de un purismo que, desde el momento mismo de la conquista y las consecutivas avanzadas imperiales, es ya imposible. Pero esto no supone una renuncia a los saberes otros, sino una apuesta a habitar las fronteras, los pliegues, y aquí es donde aparece una tercera metáfora que es la del desprendimiento, y la invitación a

(...) desprenderse de las reglas imperiales (en el conocimiento, la política, la subjetividad, etc.) y re-organizarlas en cosmologías que provienen de memorias y categorías de pensamiento ajenas a la cosmología griega, a la cosmología cristiana y a la cosmología secular de Occidente a partir de la Ilustración (2008:252).

Este desprendimiento epistémico nos habilita la apertura, el encuentro con esa exterioridad velada, o producida como ausente, tal como De Sousa Santos (2006) nos sugiere.

Aquí enlazamos con la apuesta de la Epistemología del Sur, a la renovación y a la reinvención que nos propone el portugués. Una investigación otra es una experiencia que se deslinda de la agenda de la "innovación científica" de los centros hegemónicos y liberales de la producción académica. No se trata de ofertar en el mercado académico nuevas y vistosas estrategias, sino de hacer un movimiento radical de deconstrucción que nos permita ver, sentir, pensar y hacer de una forma otra, desprendida de las lógicas hegemónicas del pensamiento moderno colonial-androcéntrico. Implica cuestionamiento de los propios privilegios, y un trabajo colectivo de destejer la trama de la colonialidad del ser que nos hace autómatas del repertorio de una epistemología racista y misógina.

La reinvención supone en nuestra mirada un mudarse a la exterioridad "en el preciso sentido del afuera (bárbaro, colonial) construido por el adentro (civilizado, imperial)" (Mignolo, 2008:252). Ese afuera se dibuja en nues- 
tra experiencia por el paisaje de las denominadas por Grüner (2016) "teorías críticas periféricas". Esas formaciones discursivas y experienciales que interpelan el centro de las teorías hegemónicas en las que hemos sido formades.

En esta línea, no podemos dejar de pensar la investigación otra como una apuesta de los feminismos situados. Lo otro por definición es para nosotres, el afuera del adentro patriarcal (androcentrado, hetero-cis-centrado y adultocéntrico). La figura del pater en el campo académico ha empobrecido (si no impedido) gestar conocimientos con potencia liberadora. La razón metonímica y proléptica (De Sousa Santos, 2003) del pensar moderno, es una razón patriarcal. No se trata sólo de las posibilidades que las mujeres tenemos de trabajar y producir en la academia. Se trata de las posibilidades del conocimiento de transformar el mundo. Desarmar las conexiones entre diversas teorías (liberales o críticas) y diversas formas de actuación del patriarcado es parte de la agenda de una investigación otra. Tal como Millan (2014) apunta:

En el centro de nuestras discusiones ha estado el cómo elaborar un pensamiento propio que acepte conceptos y teorías preexistentes y que, al mismo tiempo, abra el espacio para pensar lo nuevo, desde nuestras localidades y espacios sociales de acción y de investigación, apto para enunciar lo que no ha sido dicho porque nos faltan las palabras o porque no se escuchan las voces subalternizadas de lo femenino en plural (2014:11).

Lo otro, entonces, es lo nuestro. Es un acto de liberación y justicia biográfica, histórica y epistémica, porque nos permite conjurar la alienación de la que ya nos hablaba Dussel (1994), cuando denunciaba cómo Europa se configuró como lo Mismo en diferencia de un Otro al cual subalternizar. Lo otro, como apunta Carballeda (2017) es lo nuestro capturado por el discurso eurocéntrico que nos hace ser extranjeres en nuestra propia tierra, que nos impone la sumisión, la indefensión, el desconocimiento y desprecio por nuestros legados.

Una investigación otra es emancipadora, porque va por el camino de la descolonización, la despatriarcalización, la conexión con nuestro deseo, la colectivización de nuestras demandas, la invención de formas otras de ser, estar, soñar, escribir, investigar, intervenir. 


\section{Inscripciones curriculares}

Somos conscientes de que nos insertamos en un campo de extensa trayectoria. Los estudios curriculares tienen toda una tradición, que se destaca entre otras cosas por su reflexividad. Al campo de la investigación cualitativa le debemos no pocas herramientas metodológicas que hicieron allí su prueba de fuego para migrar luego al acervo de otros campos de estudio. Experiencias como la de investigación-acción, el docente como investigador de su propia práctica, los enfoques etnográficos en educación, e incluso la propia pedagogía decolonial, nos muestra la diversidad y productividad de un campo dinámico que no deja de reinventarse. A su vez, nos llama la atención pensar que es un campo que convoca a la interdisciplina. Los estudios sobre curriculum lo son sobre campos de formación, de disciplinas puntuales pero diversas. Ese cruce deja marcas. No puede homologarse una investigación sobre la formación de profesionales de la medicina, la matemática, las letras o del Trabajo Social mismo. Tampoco son experiencias inconmensurables entre sí. Pero esos cruces producen efectos, ponen en jaque los propios "sentidos comunes" que cada campo contiene y reproduce, y en ese movimiento favorecen la apertura de transformaciones más que interesantes.

En nuestro caso, al intersectar el campo del Trabajo Social, con el campo de las teorías del curriculum y las ciencias de la educación, y el campo de los debates de pensar situado (incluyendo allí diversas perspectivas que abonan a la crítica de la colonialidad patriarcal moderna heterociscentrada), encontramos que ninguno de estos tres grandes locus podía salir "indemne". Había interpelaciones cruzadas que hacen que nuestros primeros interrogantes, del tipo “¿qué se enseña sobre determinado tema en Trabajo Social?”, deban recorrer un largo camino de clarificación teórica y metodológica para devenir problemas de investigación justificados, comprensibles, factibles, relevantes y con capacidad heurística para ser traducidos en un trabajo de campo que combine sistematicidad, creatividad y posibilidad de realización.

Preguntarse qué se enseña, nos remite a preguntarnos previamente qué es enseñar, cual es el estatuto de la relación entre el enseñar y el aprender, a qué llamamos formación y como esta dialoga con los otros dos conceptos; quién enseña, cuándo y dónde, cómo se enseña, si lo que se enseña es cons- 
titutivo o no respecto del cómo se lo enseña, qué ocurre con lo que no se enseña, si lo no enseñado es parte (en tanto lo invisible es producido activamente como tal, según nos explica De Sousa Santos (2010), donde quedan registrados los vestigios de lo que se enseña cuando no se está enseñando oficialmente, qué distancias y articulaciones hay entre lo que se dice que se va a enseñar y lo que efectivamente se enseña, cuál de estas dos cuestiones podemos relevar, qué valor tiene cada una de ellas, etc.

De este nudo problemático surge la noción que aquí queremos presentar: inscripciones curriculares. Es el resultado del largo viaje al que estas preguntas nos llevaron, y que recoge experiencias concretas de investigaciones previas y propuestas de investigaciones por venir. Es una brújula, es una cartografía, es un barco. Nos permite saber a dónde vamos, nos permite reconocer diferentes caminos posibles para lidiar con las incomodidades sin eliminarlas, nos brinda un medio de locomoción y avance.

Hablamos entonces de inscripciones curriculares para referirnos a un conjunto de artefactos discursivos que circulan en la trama curricular, y que de manera performativa producen acciones, diagraman posiciones y delinean posibilidades en los procesos formativos. Como hemos apuntado previamente, lo curricular desborda la dimensión prescriptiva del plan de estudios, y se mueve en un conjunto de procesos que abarcan instancias diversas como son el clima institucional, las formas de resolver la relación docente-estudiante, las articulaciones de la docencia con la investigación y la extensión, los modelos evaluativos, etc. Esta mirada dinámica hace compleja la tarea de objetivar qué es lo curricular, dónde está, cómo verlo, identificarlo, cualificarlo, analizarlo, y finalmente transformarlo. Existe una dimensión elusiva del curriculum a pesar de ser un objeto de peso propio y bien documentado. Y esto es así porque la dimensión explícita de lo curricular es tan pregnante, tan asible, tan dispuesta a ser inspeccionada que su lado b, sus elementos nodales que se juegan en lo tácito, lo implícito, lo no enseñado (lo que aquí denominamos currículum implícito y nulo), quedan sistemáticamente invisibilizados. Nuestras tradiciones investigativas, profundamente influenciadas por el pensamiento positivo moderno, como hemos dicho en 
escritos previos citando a De Sousa Santos (2010), se llevan mal con el estudio de lo ausente, lo no dicho, lo silente, lo mutable, lo no estable.

Queremos proponer una idea de inscripción otra. Una inscripción no necesariamente escritural, aunque sí discursiva, en los términos que la noción de discurso en Foucault (1992), y en Angenot (2010) nos permiten. Pensar lo curricular en términos de inscripciones curriculares nos permite reconocer en la superficie aparentemente llana del por momentos remanido discurso de la didáctica moderna, la emergencia de nudos, texturas, disrupciones, pequeñas alteraciones. Son esas irrupciones las que nos convocan a la escucha.

Investigar inscripciones curriculares es entonces, agudizar nuestros sentidos y ampliar el campo de estudio. Esto supone diferentes cuestiones. Por un lado, nuestro objeto de investigación puede verse diversificado en su composición. A las clásicas unidades de información como son el plan de estudio, los programas y los propios sujetos del acto educativo (docentes, estudiantes, gestión), se suman otros artefactos donde pueden encontrarse vestigios de estas inscripciones. Hablamos de los afiches y murales que pueblan nuestros pasillos universitarios, hablamos del acta de una sesión de consejo académico, hablamos de un mensaje de whatsapp. Estos territorios otros, excluidos de las propuestas tradicionales de investigación por su carácter espinoso, difícil de ser abarcado en un universo o muestra, pueden ser sin embargo espacios-tiempos determinantes en los procesos de producción y reproducción curricular.

Entonces aquí viene nuestro segundo señalamiento. $\mathrm{Y}$ es que, si bien la noción de inscripciones curriculares amplía enormemente las superficies donde puede visualizarse la complejidad de los procesos formativos que nos ocupan, también en el mismo movimiento, focaliza abruptamente esa masa de potencial información, desde un criterio preciso y operativo de pertinencia que hace a esta idea potente no solo en términos de densidad epistémica sino de factibilidad. Porque las inscripciones curriculares son inscripciones de algo. Son esos artefactos discursivos que se configuran en torno de un objeto que es de nuestro interés. En nuestro caso ese objeto-proceso es lo descolonial. Vemos entonces que estamos intentando aquí suturar algo que la metodología de investigación moderna ha intentado por todos los 
medios separar: unidad de análisis y variable. En esta sutura se resuelven muchos problemas que la escisión teoría-práctica nos generó. Entonces lo que investigamos no es el currículum objeto, no es el currículum y toda su inabarcable área de influencia, no es la teoría descolonial en sí: son las inscripciones curriculares de lo descolonial en un espacio muy preciso que es el de la reproducción de nuestros cuadros profesionales.

Un último beneficio que encontramos en esta herramienta conceptual que aquí proponemos, es que nos permite ensayar la apuesta a la que nos convoca Deleuze (2009) cuando advierte que en el acto de escribir no puede escindirse forma de contenido. La forma de investigación de lo descolonial no puede ser moderna-colonial. No puedo producir un saber otro de un método de lo mismo. Y a la vez, en esta zona de frontera (Anzaldúa, 1987) que es la academia, no podemos tampoco abandonar la pretensión de rigurosidad que supone intentar producir un conocimiento fundado en términos lógicos y empíricos. Quizás ese anarquismo epistemológico al que Feyerabend (1984) nos invitaba se pueda revisitar, para sacar de allí un gesto, una apuesta, que articule con la laboriosa tarea que supone el armado de un corpus, con la inventiva tarea que implica el diseño de herramientas para hacernos de los sentidos que laten en las prácticas, con la colectiva esperanza de comprender para transformar.

Inscripciones curriculares sobre Estado, el Poder y la Política en Trabajo Social

En este apartado puntualizaremos lo que fue nuestra primera experiencia de análisis de la formación en Trabajo Social (Hermida, 2016). En el marco de este estudio, comenzó a prefigurarse esta idea de inscripciones curriculares, aunque pudimos sistematizarla recientemente, luego de diversas experiencias de investigación, docencia, extensión y gestión.

En esa ocasión, el objetivo de la investigación fue el de analizar los discursos en torno al Estado, el Poder y la Política en la formación de grado en Trabajo Social. Del estado del arte construido para esa ocasión, lo que emergió como constatación, es que, si bien la formación académica de la disciplina era objeto privilegiado de debates y publicaciones no había dis- 
ponibles estudios que tomaran como universo de estudio la formación de grado del país. Es decir, que las producciones privilegiaban el debate teórico. Y aquellas que incluían análisis empírico (relevamiento de planes de estudio, programas, trabajo de campo con estudiantes, docentes o graduades) tomaban como objeto a universos más acotados (una carrera, una provincia, una región).

La otra peculiaridad de ese estudio, cuando lo revisitamos desde este presente, es que construimos el mismo en momentos previos a la emergencia de los profesorados en Trabajo Social y la expansión de carreras posgraduales como las especializaciones en docencia universitaria, que se fueron creando y ampliando con los años, promoviendo nuevos estudios que pusieran en foco la discusión de qué enseñamos y cómo lo hacemos en Trabajo Social

La apuesta a expandir el universo de unidades de análisis, nos confrontó con el desafío de focalizar y seleccionar con precisión qué de la formación habríamos de relevar. Fue así que el foco estuvo puesto en los debates en torno del Estado, el Poder y la Política. Cabe mencionar que este plan de trabajo fue concebido en 2010. Aún estaba muy presente en nuestras preocupaciones la "ruptura epistemológica (Bachelard, 2004) e interventiva que supuso el modelo de desarrollo kirchnerista y su concomitante reestructuración de la política social argentina. Esos procesos, iniciados en 2004 de la mano de la reinvención del Ministerio de Desarrollo Social de la Nación por parte de la entonces ministra Alicia Kirchner, hicieron mella en nosotras, las colegas graduadas en la década del noventa, donde la formación apuntaba a una crítica del Estado que por definición se significaba como neoliberal, siempre parte del problema, y nunca parte de la solución.

De esta forma, la tesis buscaba contrastar la hipótesis de que lo que hoy denominamos las inscripciones curriculares en torno del Estado, el Poder y la Política estaban o bien minimizadas e invisibilizadas, o bien connotadas en el marco de una sub-línea del enfoque marxista, denominada histórico-crítica (Martinelli, 1992) que significaba al Estado como escribanía de la burguesía, discriminando entre proyectos revolucionarios o reformistas (Montaño, 2004), incluyendo en este segundo grupo a todos los gobiernos del denominado giro a la izquierda en la región (Pérez, 2011). Lo que conje- 
turamos allí era la "presencia de una ausencia", y esa ausencia era la de teorías posestructuralistas, neomarxistas, agonistas, latinoamericanas, situadas y descoloniales, como marcos explicativos otros que permitieran una mirada diferente para comprender, habitar y transformar el Estado.

Durante el desarrollo de este trabajo de investigación, fue creciendo nuestro interés por el locus pos/des colonial. Entonces, la tesis, también aborda las presencias y ausencias de inscripciones curriculares de la crítica de lo colonial y del feminismo, pero de manera incipiente, como una de las teorías a revisar en sus alcances para nuestra formación.

En ese momento no lo sabíamos, pero hoy sí. Esa tesis fue una de las diversas puertas de entrada a una estrategia colectiva de construcción del problema de la crítica de lo racial, lo sexo-genérico, lo disidente, lo nuestroamericano, lo situado, en el campo de la investigación en Trabajo Social. En ese recorrido, es que emerge nuestra segunda experiencia.

Inscripciones curriculares en torno a lo descolonial en Trabajo Social

Como mencionamos anteriormente, otra de las experiencias que nos convocan en este artículo presenta la pregunta de cómo se inscribe curricularmente lo descolonial en la formación de grado de Trabajo Social en nuestro país, optando por indagar este aspecto desde los aportes del "giro descolonial".

Esta decisión se remonta a la experiencia de investigación de grado (Tiberi, 2018), en la cual se indagó la presencia y las posibilidades de un pensamiento crítico de y para Latinoamérica que pueda representar un aporte en la construcción y el desarrollo de procesos de formación crítica en Trabajo Social. El recorrido emprendido atravesó las teorías y producciones que marcaron la agenda intelectual latinoamericana, en sintonía con los procesos sociales y políticos más radicalizados. En esa búsqueda, se concretó el encuentro con las producciones del giro decolonial impulsadas desde el grupo latinoamericano modernidad/colonialidad, que emergieron como propuestas potentes para proyectar la construcción de ese pensar crítico. A partir de allí, lo descolonial comenzó a perfilarse como una perspectiva posible. 
Aunque no fue el objetivo inicial, en dicho trabajo se pudo advertir la ausencia relevante del enfoque descolonial en las propuestas de formación del Trabajo Social. Este aspecto quedó resonando, haciendo eco. Sembró una pregunta, un interrogante, ese que, como decíamos, nos mueve y moviliza... ¿Por qué lo descolonial no estaba presente en la formación? ¿Qué está pasando en otras unidades académicas? ¿También está ausente? Y si está presente... ¿De qué manera? ¿Con qué producciones? De la semilla de estas preguntas se fue gestando el proyecto de investigación ${ }^{1}$.

Allí, los interrogantes se fueron encaminado a conocer la presencia de lo descolonial en la formación de grado de Trabajo Social en el país. Un primer ejercicio fue, entonces, delimitar el aspecto de la formación a indagar. Con el interés puesto en las propuestas de formación, se delimitó el aspecto de la enseñanza, enfocando el interés en el currículum, en los documentos que rigen, organizan y regulan el desarrollo de las carreras y las asignaturas que lo conforman. En ese punto se materializa la intersección de los campos mencionados: el Trabajo Social, las teorías del currículum y las ciencias de la educación, y el campo de los debates de pensar situado, en este caso de lo descolonial. Las contribuciones de Hermida $(2016,2014)$ fueron alumbrando el camino e invitando a ensayar esta intersección, este encuentro potente entre los campos de estudio.

En este punto se ubicó el desafío antes mencionado, de no escindir la forma del contenido en la investigación. Parafraseando a Mignolo (2007), fue una apuesta de desprendimiento de los marcos modernos/coloniales y apertura a formas otras de producir ese conocimiento. $Y$ es que la crítica descolonial denuncia no sólo las pretensiones universalizantes del discurso moderno/colonial, sino también sus límites y su lógica "epistemicida" que niega y extermina saberes y metodologías otras por no considerarlas válidas. Cómo aspirar entonces a generar conocimientos sobre lo descolonial sin abrirnos antes a formas otras de producirlos, sin que esto implique el

1 El proyecto mencionado fue elaborado en vistas a la postulación para las Becas Internas Doctorales 2021 impulsadas por el Consejo Nacional de Investigaciones Científicas y Técnicas de Argentina (CONICET). 
abandono de las potentes máquinas de pensar que nos ofrece el discurso moderno (Hermida, 2015). Aspirar, entonces, a una ecología de saberes que nos permita su diálogo (De Sousa Santos, 2010).

En esta línea, se articularon y triangularon distintas corrientes epistémicas, apostando a un diálogo entre sí, para construir el marco del proyecto. Los aportes de la crítica descolonial conformaron el nudo conceptual fundante, dando un lugar principal a los desarrollos acerca de la problemática educativa (Mouján, 2014) así como de las pedagogías descoloniales (Walsh, 2013). En la misma línea, se ensayaron articulaciones que permitieron enriquecer el abordaje teórico-metodológico convocando al diálogo aportes de la teoría del currículum, centralmente desde las contribuciones de Eisner (1979). Allí fue emergiendo y encontrando su lugar, la categoría de inscripciones curriculares.

Si bien se destaca la importancia de las contribuciones del giro descolonial en los procesos de formación que nos convocan, se reconocen los límites que desde esta perspectiva se presentan sobre la ausencia o no profundización de algunas líneas de análisis de importancia, como la de los feminismos. En este punto se refuerza la importancia de los trabajos mencionados, fundamentalmente la línea de investigación emprendida por Roldán (2020).

Inscripciones curriculares de los Feminismos del Sur en Trabajo Social

La incipiente construcción colectiva de la categoría inscripciones curriculares, además de gestarse en las investigaciones anteriormente expuestas, se vio atravesada por otra investigación en la que problematizamos el pensar situado, a partir de la constelación de saberes de los Feminismos del Sur, en la formación de grado en Trabajo Social en la Universidad Nacional de Mar del Plata, durante el ciclo académico 2018. En esa instancia, la inquietud estuvo puesta en conocer qué de los Feminismos del Sur se explicitaba en la currícula de dicha licenciatura. En esta dirección, optamos por recuperar algunos aspectos del abordaje teórico, epistemológico y metodológico que Hermida (2016) realizó, estableciendo un punto de partida y un modo par- 
ticular de acercarnos a la indagación de este campo problemático y proponiendo una forma de ver y teorizar la formación de grado en Trabajo Social.

Específicamente, este proceso cognitivo implicó develar qué saberes, contenidos, sobre los Feminismos del Sur circulaban, durante ese periodo, en la oferta académica en el currículum explícito (Eisner, 1979). A ese marco lo enlazamos con aspectos teóricos-epistemológicos de la sociología de las ausencias (De Sousa Santos, 2008) al comprender que aquellos saberes que fueron omitidos y/o descartados fueron producidos como ausentes en el marco de una modernidad colonial patriarcal. El interés que justificó la indagación se fundó en la premisa de que los Feminismos del Sur, como aquellos loci de enunciación que tienden a subvertir el orden social (Roldán, 2020), se constituyen como una perspectiva política, epistémica y metodológica indispensable para la formación de trabajadores sociales.

Del mencionado proceso de investigación arribamos a diferentes cuestiones. Por un lado, los contenidos sobre los Feminismos del Sur ocuparon un lugar mínimo en el plan de estudios y los programas docentes, de manera contraria, hubo una priorización de conceptos, categorías y aportes del enfoque histórico crítico. Por otro lado, esta ausencia de conocimientos, que los saberes de los Feminismos del Sur nos proveen, se vio tamizada al vislumbrar en las percepciones de docentes y estudiantes una mayor presencia e interés por tratarlos e incrementar su inclusión en la formación de les estudiantes. Por lo tanto, aunque estos feminismos no formaran parte explícita del plan de estudios y los programas docentes, evidenciamos un naciente interés académico compartido por docentes en gestión, docentes y estudiantes de problematizar y transversalizar los feminismos en general y los Feminismos del Sur en particular en cada escenario pedagógico.

Ahora bien, el recorrido emprendido se complejizó y tomó densidad, por lo que, necesariamente, las preguntas se corrieron y expandieron ¿Esta particular manera de interacción de los feminismos en la formación de grado en Trabajo Social, en la UNMDP, comparte con, y entre ellas, regularidades con el resto de las unidades académicas de la Argentina? Si los feminismos estuviesen presentes en el currículum de esas universidades nacionales $\dot{i}$ En 
cuáles dispositivos se inscriben? ¿Y a qué locus/lugar de enunciación corresponden dichas inscripciones curriculares?

Con base en esas preguntas, decidimos ampliar la dimensión espacial, de pasaje de estudio de una particular unidad académica, a la re-construcción de una muestra (Hermida, 2016) que abordara el fenómeno a nivel nacional, en Argentina. En esta búsqueda vital por profundizar la comprensión de la interacción de los feminismos situados en la formación profesional, es que el concepto-herramienta de inscripciones curriculares adquiere alta relevancia. De ahí que, en la construcción de la reciente provocación, reflexionamos acerca de que, los feminismos no solo se imprimen en los documentos curriculares, sino que se tallan en múltiples aristas que conforman la propuesta pedagógica de cada unidad académica. En este sentido, un reto a contemplar alude a la estrategia analítica, de no solo referenciar las matrices teóricas y epistemológicas en las que fundan los planes de estudios y programas docentes, sino explicitar los artefactos discursivos que propician la inscripción de los Feminismos del Sur en la currícula. Es decir, que la dimensión de estudio, abarca a todos aquellos dispositivos pedagógicos que de manera directa o indirecta disponen la apropiación de los conocimientos y saberes sobre los feminismos y que inciden en las propuestas y procesos formativos, como la relación que se establece entre las funciones de la universidad de docencia-investigación-extensión y las políticas de género y cuidado que cada unidad académica, en el marco de un proyecto político-académico, adopta.

Por lo tanto, lo hasta aquí expuesto, remite a visibilizar los procedimientos operativos que empleamos para aproximarnos al cruce de los feminismos situados y la formación de les estudiantes en Trabajo Social. Un cruce novedoso, dadas las tradiciones que conjugan en él, que hace distintiva la forma de abordar el problema de la formación profesional y que se anuda a otros dos que son los propuestos por Tiberi (2018) y Hermida (2016) para la co-construcción de lo que, en esta comunicación, nombramos como inscripciones curriculares. 


\section{Conclusiones}

Las experiencias presentadas intentan reconstruir algo de ese largo y profundo viaje en el que nos hemos embarcado desde hace algunos años y que nos permitió cosechar esta noción, esta nueva propuesta para investigar y conocer sobre la formación: las inscripciones curriculares. Un artefacto que se incorpora a nuestra caja de herramientas y que busca abrirse un lugar en el acervo epistemológico y metodológico de la investigación en Trabajo Social.

Esta presentación, este anuncio, es también una invitación a seguir pensando y reflexionando sobre el ejercicio de la investigación, a observar con una mirada crítica las reglas modernas y coloniales que rigen hoy el universo de la producción de conocimiento para, a partir de allí, ensayar movimientos que nos permitan desprendernos y abrirnos hacia formas otras de conocer.

También es una apuesta a intersectar diversas áreas disciplinares, como son las ciencias de la Educación, el Trabajo Social, los Estudios de Género, la Epistemología, intentando trascender las posiciones instrumentalistas y la sobre especialización, ensayando formas de habitar el campo y de trabajar que sean respetuosas de los procesos que buscamos comprender y de las premisas que intentamos sostener. Más que proponer crear sub-campos sobre-especializados dentro de una disciplina, buscamos construir puentes para movernos en el espacio que nuestras preguntas bordean.

Reconocemos las tensiones y dificultades que se presentan en la intersección de estos campos de estudio. Las reconocemos, pero también las habitamos, las atravesamos. Nos mueve la certeza de superar los límites palpables del modus operandi clásico moderno/colonial/patriarcal apostando a la potencia de lo descolonial y de los feminismos del sur.

\section{Referencias bibliográficas}

Angenot, Marc (2010). El discurso social. Los límites de lo pensable y lo decible. Buenos Aires, Argentina, Siglo XXI editores.

Anzaldúa, Gloria (1987). Borderlands/La Frontera: The New Mestiza. San Francisco, EEUU, Ediciones Aunt Lute.

Bachelard, Gastón (2004). La formación del espiritu científico. México, Siglo XXI. 
Carballeda, Alfredo (2017). "La negación de lo Otro como violencia. Pensamiento de-colonial y cuestión Social”. En M. E. Hermida y P. Meschini (Ed.), Trabajo Social y Descolonialidad (pp. 67-78). Mar del Plata, Argentina, EUDEM.

Deleuze, Gilles (2009) Crítica y clinica. Barcelona, España, Editorial Anagrama.

De Oto, Alejandro y Alvarado, Mariana (2017). “Junturas". En A. De Oto. (Ed.), Metodologías en contexto. Intervenciones en perspectiva feminista/poscolonial/ latinoamericana (pp. 9-12). Buenos Aires, Argentina, CLACSO.

De Sousa Santos, Boaventura (2006). Renovar la teoría crítica y reinventar la emancipación social. Buenos Aires, Argentina, CLACSO.

De Sousa Santos, Boaventura (2008). Conocer desde el Sur. Para una cultura política emancipatoria. Buenos Aires, Argentina, CLACSO.

De Sousa Santos, Boaventura (2010). Descolonizar el saber, reinventar el poder. Montevideo, Uruguay, Ediciones Trilce.

Dussel, Enrique (1994). 1492: el encubrimiento del otro : hacia el origen del mito de la modernidad. La Paz, Bolivia, Plural Editores. Recuperado de: http://biblioteca.clacso.edu.ar/clacso/ otros/20111218114130/1942.pdf

Eisner, Elliot (1979). The educational imagination: on the design and evaluation of school programs. New York, EEUU, EDEL.

Feyerabend, Paul (1984). Adiós a la razón. Madrid, España, Tecnos.

Foucault, Michel (1992). El orden del discurso. Buenos Aires, Argentina, Tusquets editores.

Grüner, Eduardo (2016). “Teoría Crítica y Contra-modernidad. El color negro: de cómo una singularidad histórica deviene en dialéctica crítica para «nuestra América», y algunas modestas proposiciones finales". En J. Gandarilla Salgado (Ed.), La crítica en el margen: Hacia una cartografia conceptual para rediscutir la modernidad. Buenos Aires, Argentina, AKAL.

Hermida, María Eugenia (2014). El curriculum que prescribe y que proscribe. Por una didáctica de las ausencias en Trabajo Social. Revista de Educación, (7), 327-345. https://fh.mdp.edu. ar/revistas/index.php/r educ/article/download/996/1037

Hermida, María Eugenia (2015). Colonialismo y producción de ausencias. Una crítica desde el Trabajo Social para visibilizar los presentes subalternos. Debate Público. Reflexión de Trabajo Social, 5(10), 67-85. http://trabajosocial.sociales.uba.ar/wp-content/uploads/sites/13/2016/03/09 Hermida.pdf

Hermida, María Eugenia (2016). Discursos sobre estado, poder y política en la formación de grado en trabajo social. (Tesis de Doctorado) Universidad Nacional de Rosario, Rosario, Argentina.

Martinelli, María Lucia (1992). Servicio Social: Identidad y alienación. Sao Paulo, Brasil, Cortez Editora.

Mignolo, Walter (2007). "El pensamiento des-colonial, desprendimiento y apertura: un manifiesto”. En S. Castro-Gómez, y R. Grosfoguel (Ed.), El giro decolonial. Reflexiones para una diversidad epistémica más allá del capitalismo global (pp. 25-46). Bogotá, Colombia, Siglo del Hombre.

Mignolo, Walter (2008). La opción de-colonial: desprendimiento y apertura. Un manifiesto y un caso. Tabula Rasa, (8), 243-281. http://www.revistatabularasa.org/numero-8/mignolo1. pdf 
Millán, Márgara (2014). Más allá del feminismo: caminos para andar. México D.F., México, Red de Feminismos Descoloniales. Recuperado de: https://radiozapatista.org/wp-content/ uploads/2018/03/Mas-alla-del-feminismo.pdf

Montaño, Carlos (2004). Hacia la construcción del Proyecto Ético-político Profesional crítico. XVIII Seminario Latinoamericano de Escuelas de Trabajo Social ALAETS Costa Rica. Recuperado de: http://www.ts.ucr.ac.cr/binarios/congresos/reg/slets/slets-018-045.pdf

Mouján, Inés (2014). Miradas descoloniales en la educación. Intersticios de la política y la cultura. Intervenciones latinoamericanas, 3(6). https://revistas.unc.edu.ar/index.php/intersticios/ article/view/9898/10741

Ortiz Ocaña, Alexander; Arias López María Isabel y Pedrozo Conedo, Zaira (2018). Metodología 'otra' en la investigación social, humana y educativa. El hacer decolonial como proceso decolonizante. Revista FALA, 7(30), 172-200.

Pérez, Germán (2011). Todo aquel fulgor. La politica argentina después del Neoliberalismo. Buenos Aires, Argentina, Nueva Trilce.

Rivas Flores, José I. (2020). Una investigación “otra”, para una educación “otra”, para una sociedad "otra". Hegoa, 9, 26-27.

Rivera Cusicanqui, Silvia (2018). Un mundo ch'ixi es posible. Ensayos desde un presente en crisis. Ciudad de Buenos Aires, Argentina, Tinta Limón.

Roldán, Yanina, (2020). Los Feminismos del Sur en la formación de grado en Trabajo Social, 2018 (Tesis de grado). Universidad Nacional de Mar del Plata, Mar del Plata, Argentina.

Roldán, Yanina. (2020). Los Feminismos del Sur en la formación de grado de Trabajo Social. Millcayac - Revista Digital De Ciencias Sociales, 7(13), 575-588. http://revistas.uncu.edu.ar/ ojs/index.php/millca-digital/article/view/2588

Tiberi, Renzo (2018). El Trabajo Social por la senda del pensamiento crítico latinoamericano: aportes para la construcción de un pensamiento crítico en nuestra formación (Tesis de grado). Universidad Nacional de Rosario, Rosario, Argentina.

Walsh, Catherine (2013). Pedagogias decoloniales: prácticas insurgentes de resistir, (re) existiry (re) vivir. Quito, Ecuador, Abya Yala.

Recibido: 10/02/2021

Aceptado: 26/04/2021 